\title{
NUMERICAL INVESTIGATION OF BOUNDARY LAYERS IN WET STEAM NOZZLES
}

\author{
Jörg Starzmann;, Fiona R. Hughes and Alexander J. White \\ Hopkinson Laboratory, Department of Engineering, University of Cambridge \\ Cambridge, United Kingdom \\ Marius Grübel and Damian M. Vogt \\ ITSM, University of Stuttgart \\ Stuttgart, Germany
}

\section{ABSTRACT}

Condensing nozzle flows have been used extensively to validate wet steam models. Many test cases are available in the literature and in the past a range of numerical studies have dealt with this challenging task. It is usually assumed that the nozzles provide a one- or two-dimensional flow with a fully turbulent boundary layer. The present paper reviews these assumptions and investigates numerically the influence of boundary layers on dry and wet steam nozzle expansions.

For the narrow nozzle of Moses and Stein it is shown that the pressure distribution is significantly affected by the additional blockage due to the side wall boundary layer. Comparison of laminar and turbulent flow predictions for this nozzles suggests that laminar-turbulent transition only occurs after the throat. Other examples are the Binnie nozzle and the Moore nozzles for which it is known that sudden changes in wall curvature produce expansion and compression waves that interact with the boundary layers. The differences between two- and three-dimensional calculations for these cases and the influence of laminar and turbulent boundary layers are discussed.

The present results reveal that boundary layer effects can have a considerable impact on the mean nozzle flow and thus on the validation process of condensation models. In order to verify the accuracy of turbulence modelling a test case that is not widely known internationally is included within the present study. This experimental work is remarkable because it includes boundary layer data as well as the usual pressure measurements along the nozzle centreline. Predicted and measured boundary layer profiles are compared and the effect of different turbulence models is discussed. Most of the numerical results are obtained with the in-house wet steam RANS-solver, Steamblock, but for the purpose of comparison the commercial program ANSYS CFX is also used, providing a wider range of standard RANS-based turbulence models.

\section{NOMENCLATURE}

$c_{\mathrm{p}} \quad$ specific heat capacity at constant pressure $\left[\mathrm{J} \mathrm{kg}^{-1} \mathrm{~K}^{-1}\right]$

$E \quad$ internal energy $[\mathrm{J}]$

$J \quad$ nucleation rate $\left[\mathrm{kg}^{-1} \mathrm{~s}^{-1}\right]$

$\mathrm{k}_{\mathrm{B}} \quad$ Boltzmann's constant $\left[\mathrm{JK}^{-1}\right]$

Kn Knudsen number [-]

$L \quad$ latent heat $\left[\mathrm{J} \mathrm{kg}^{-1}\right]$

$m_{\mathrm{m}} \quad$ mass of a water molecule $[\mathrm{kg}]$

Ma Mach number $[-]$

$\dot{P} \quad$ expansion rate $=-(1 / p) \mathrm{d} p / \mathrm{d} t\left[\mathrm{~s}^{-1}\right]$

$p \quad$ pressure $[\mathrm{Pa}]$

Pr Prandtl number $[-]$

$q_{\mathrm{c}} \quad$ condensation coefficient $[-]$

$r \quad$ radius $[\mathrm{m}]$

$r_{32}$ Sauter mean droplet radius [m]

*corresponding author. js2145@cam.ac.uk 
$\mathrm{R} \quad$ specific gas constant $\left[\mathrm{J} \mathrm{kg}^{-1} \mathrm{~K}^{-1}\right]$

$\mathrm{Re} \quad$ Reynolds number [-]

$t \quad$ time [s]

$T$ temperature $[\mathrm{K}]$

$V \quad$ velocity $\left[\mathrm{m} \mathrm{s}^{-1}\right]$

$x, y, z \quad$ Cartesian coordinates

$x, \theta, r \quad$ Cylindrical coordinates

$l, b, h$ length, depth, height [m]

\section{Greek Symbols}

$\alpha \quad$ droplet growth factor of Young [1] [-]

$\gamma \quad$ specific heat ratio $[-]$

$\phi \quad$ Kantrowitz isothermal correction $[-]$

$\lambda$ conductivity $\left[\mathrm{W} \mathrm{m}^{-1} \mathrm{~K}^{-1}\right]$

$\theta \quad$ momentum thickness [m]

$\rho$ density $\left[\mathrm{kg} \mathrm{m}^{3}\right]$

$\sigma \quad$ surface tension $[\mathrm{N} / \mathrm{m}]$

\section{Subscripts}

BL boundary layer

g gaseous

in inlet

f liquid

s saturated conditions

$\mathrm{t}$ stagnation conditions

crit critical conditions

\section{INTRODUCTION}

The numerical prediction of condensing steam flow in steam turbines is still a challenging and demanding task. Although one- and two-dimensional methods have been available for many years (e.g. [1-3]) and several three-dimensional CFD codes are now emerging (e.g. [4-7]), steam turbine designers have not fully incorporated wet steam flow calculations into their design process. There are several reasons why this might be so, but it is sometimes argued that the computational methods have not yet been comprehensively proven.

Wet steam models have often been tested against measurements of condensing flow in rectangular cross-section nozzles. In particular, several test cases have been used repeatedly to validate and calibrate nucleation and droplet growth expressions. The best-known low-pressure nozzle experiments were published by Moses and Stein [8] and Moore et al. [9] and these are especially valuable because they include both droplet size data and pressure distributions. The experimental data of Binnie and Green [10] and Barschdorff [11] are also frequently used. Compared with measurements in model or even full-size turbines, nozzle experiments are much cheaper and much less complex. Their relative simplicity is due to the lack of rotating parts and the straightforward geometry, usually comprising profiled top and bottom walls but planar side walls. The latter allow easy access to the flow, particularly for optical droplet-sizing techniques. Furthermore, careful design of the profiled walls can in theory provide an essentially one-dimensional flow, in contrast to the highly threedimensional, unsteady flow inherent in turbines.

The present paper focuses on whether it is reasonable to consider the aforementioned nozzle flows as one- (or possibly two-) dimensional. Of particular interest is the effect of the top, bottom and side wall boundary layers on the pressure distribution and their influence on the condensation process.

\section{PREVIOUS WORK}

Early attempts to compare computed and measured nozzle flows were based on one-dimensional, inviscid methods (e.g. Oswatisch [12], Young [1]) and thus adopted the simple expedient of inferring the effective nozzle area variation from the measured dry expansion pressure distribution. This provides a straightforward means of accounting for the effect of boundary layer blockage and is possibly the most reliable way of testing the nucleation and growth theories, provided the flow is one-dimensional. The two-dimensional inviscid time-marching method of White and Young [13] highlighted that Prandtl-Meyer expansion fans occur for some test cases due to profile curvature discontinuities near the throat. Famous examples are the Moore nozzles, comprising a straight wall blended with a curved converging section. Moore nozzles B and C have been further investigated by Simpson and White [14] who compared inviscid, laminar and fully turbulent calculations and pointed out that already in two-dimensions (i.e. neglecting the boundary layer on the side walls) the state of the boundary layer affects the centreline pressure and the wetness formation.

Nowadays numerical methods are often based on threedimensional Reynolds-averaged Navier-Stokes equations but, nonetheless, some recent investigations have only considered the boundary layers on the curved walls and therefore assume a quasi-two-dimensional flow (e.g $[7,15])$. The aim of the present study is to investigate the impact of side wall effects, and to discuss the influence of the state (laminar or turbulent) of all boundary layers on condensation onset. Conversely, the effect of the condensation disturbance on the boundary layer growth is also considered as this impinges on the accuracy of comparisons using the original one-dimensional methods.

In the following, the numerical methods and flow solvers are first introduced and three different nozzles are then discussed: the high expansion rate nozzle of Moses and Stein, which is well designed with no strong two-dimensional effects but has a small throat area $(10 \mathrm{~mm} \times 10 \mathrm{~mm})$; the Binnie nozzle, which has a slightly larger throat area but exhibits strong two-dimensional pressure wiggles; and the large nozzle B of Moore et al., also with strong two-dimensional effects. (The latter has nonetheless been used extensively as a test case, possibly due to its good quality droplet size data.) The predicted center line pressure distribution and wetness formation using both laminar and fully- 
turbulent three-dimensional calculations are compared to quasi two-dimensional results for all these nozzles.

The second part of the paper deals with the less well-known nozzle test case that dates back to a German publication by Gyarmathy and Meyer [16]. To the present authors knowledge, this is the only test case that includes detailed measurements of the boundary layer. In order to make this valuable experiment more accessible to the international wet steam community, details of the geometry and the experimental setup are summarized within the present paper.

\section{FLOW SOLVER STEAMBLOCK}

Most of the computations presented below have been carried out using an in-house flow solver called 'Steamblock', based on John Denton's well known 'T-Block' code. The development of this three-dimensional RANS-solver started over 20 years ago [17]. The basic idea is that viscous and heat transfer terms can be added as external source terms to the momentum and energy conservation equations. The original purpose of the code was to compute ideal gas flows in turbomachinery and thus the equations are formulated in cylindrical coordinates. The conserved variables are $\rho, \rho E, \rho V_{x}, \rho r V_{\theta}, \rho V_{\mathrm{r}}$ and are stored at grid vertices. For time integration an explicit scheme called 'scree' is used, as described by Pullan and Denton [18].

T-Block was extended by Chandler et al. [19] for nonequilibrium wet-steam flow and the resulting Steamblock has since undergone continuous development. For example, it has recently been updated based on the newest version of T-Block and, in addition to a simple mixing length model, now includes the Spalart-Allmaras turbulence model as an option. (All the Steamblock calculations presented below have used the SA-model.) The usual procedure within T-Block (and Steamblock) is to use wall functions to obtain wall shear stresses but, because of the present focus on detailed boundary layer effects, these stresses have been computed directly using very fine grids, with $y_{+}$-values below one.

In order to reduce computational time, the look-up table method of Hill et al. [20] is used for all the necessary steam properties. Tables are generated using the IAPWS-IndustrialFormulation (IF97) [21] for thermodynamic properties, together with IAPWS equations for viscosity [22], conductivity [23] and surface tension [24]. Droplet formation is modelled by the classical nucleation expression augmented by the non-isothermal correction, as discussed by Bakhtar et al. [25]. The resulting equation (per unit mass of mixture) takes the form

$$
J=\frac{q_{\mathrm{c}}}{1+\phi} \sqrt{\frac{2 \sigma}{\pi m_{\mathrm{m}}^{3}}} \frac{\rho_{\mathrm{g}}^{2}}{\rho \rho_{\mathrm{f}}} \exp \left(-\frac{4 \pi \sigma}{3 \mathrm{k}_{\mathrm{B}} T_{\mathrm{g}}} r_{\mathrm{crit}}^{2}\right)
$$

where $r_{\text {crit }}$ is the Kelvin-Helmholtz critical radius, $1 /(1+\phi)$ is the non-isothermal correction, and $q_{\mathrm{c}}$ is the condensation coefficient which is assumed to be one. Expressions for $r_{\text {crit }}$ and $\phi$ are standard and may be found with discussion in Ref. [25]. Other symbols are defined in the nomenclature.

The growth of droplets is modelled with Young's [1] equations,

$$
\frac{\mathrm{d} r}{\mathrm{~d} t}=\frac{\lambda_{\mathrm{g}}}{\rho_{\mathrm{f}} L r} \frac{\left(T_{\mathrm{s}}-T_{\mathrm{g}}\right)\left(1-\frac{r_{\text {crit }}}{r}\right)}{1+3.78(1-v) \frac{\mathrm{Kn}}{\operatorname{Pr}}}
$$

where

$$
v=\frac{\mathrm{R} T_{\mathrm{s}}}{L}\left(\alpha-0.5-\frac{2-q_{\mathrm{c}}}{2 q_{\mathrm{c}}}\left(\frac{\gamma+1}{2 \gamma}\right)\left(\frac{c_{\mathrm{p}} T_{\mathrm{s}}}{L}\right)\right)
$$

and $\alpha$ is an empirical coefficient introduced by Young in order to obtain agreement with experiments at low pressure. Previous studies with Steamblock suggest that $\alpha=11$ provides the best possible agreement for several low pressure test cases and this value has been used throughout.

The liquid droplet phase is incorporated into the flow conservation equations within Steamblock using the method of moments. This method was originally introduced by Hill [26], but detailed discussion of the moment method is provided by White et al. [27, 28], together with comparisons with other droplet spectrum modelling techniques. The moment approach is very efficient because it requires only a few physical quantities to be computed rather than tracking the formation and growth of many droplet groups. In the present case four moment equations are simultaneously solved in addition to the ordinary conservation equations, as described in Ref. [19]. The moment equations are closed by the simple but robust approach of evaluating the droplet growth rate at the surface averaged radius $r_{20}=\sqrt{\mu_{2} / \mu_{0}}$. The accuracy of this and other similar moment approaches has been investigated recently by Hughes et al. [29] who conclude that, for expansion rates between $2000 s^{-1}$ and $10000 s^{-1}$ differences with full-spectrum calculations are less than $3 \%$ for the Wilson point pressure and between $5 \%$ and $15 \%$ for the average droplet diameter.

The accuracy of calculations for condensing steam flow is likely to depend mainly on the four above-mentioned issues: steam table properties, nucleation and droplet growth modelling, and the treatment of the droplet size distribution. Unfortunately a level of uncertainty and approximation is attached to each of these, particularly the nucleation and droplet growth theories. The purpose of the present paper is not however to validate (or calibrate) these aspects of wet steam modelling and thus they are not given further attention. (In this respect an "International Wet Steam Modelling Project" is currently under way to investigate 


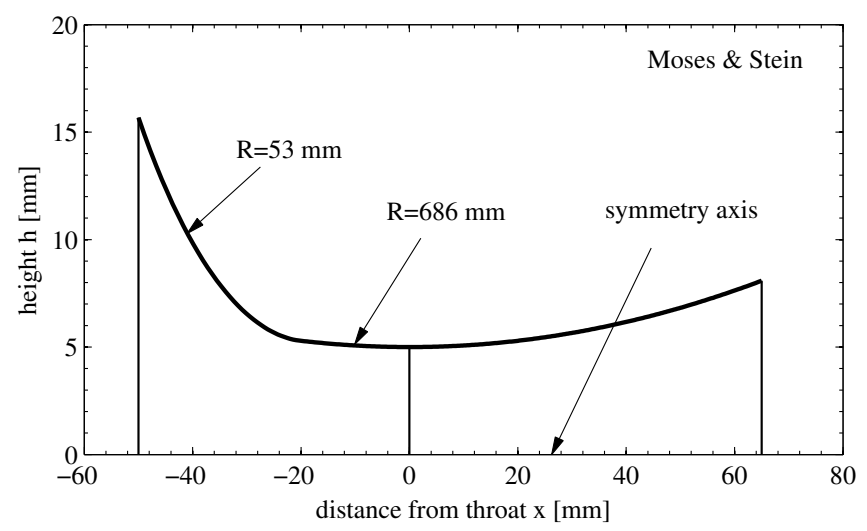

FIGURE 1. Modelled geometry for the Moses and Stein [8] nozzle

and review the current state of wet steam modelling, results from which can be expected soon [30].)

\section{ANSYS CFX}

Turbulence modelling within Steamblock is currently restricted to mixing length or the one-equation Spalart-Allmaras models. Additional calculations have therefore been carried out with the commercial solver Ansys CFX 16.2, enabling comparisons with a broader range of turbulence models. Ansys CFX is a general purpose flow solver capable of solving various types of fluid dynamic problems. The implicit coupled solver is pressure based and solves the unsteady three-dimensional NavierStokes equations. The steam properties are also based on the IAPWS-IF97 formulation by means of tables for a user-defined pressure and temperature range [21]. More information can be found in [31]. Although an Euler-Euler non-equilibrium steam model is available in CFX it is not considered to be appropriate for the present work to compare condensing steam flows. This is because differences exist not only in the numerical schemes but also in the representation of the droplet phase. (Ansys CFX uses a mono-dispersed droplet model whereas Steamblock uses the method of moments.)

\section{MOSES AND STEIN NOZZLE}

As previously mentioned, the Moses and Stein [8] nozzle is a small and narrow nozzle. The constant depth of the nozzle is only $10 \mathrm{~mm}$ and, as shown in Fig. 1, the throat height is also only $10 \mathrm{~mm}$. The geometry leads to a high expansion rate of $\dot{P}=6500 \mathrm{~s}^{-1}$ at the throat, increasing continuously downstream. In the region where condensation occurs expansion rates between $9000 \mathrm{~s}^{-1}$ and $10000 \mathrm{~s}^{-1}$ can be expected.

In order to resolve the boundary layer the numerical grid has to be very fine and it was found that a grid size of $350 \times 91 \times 91$ nodes was required for grid-independent solutions. The test

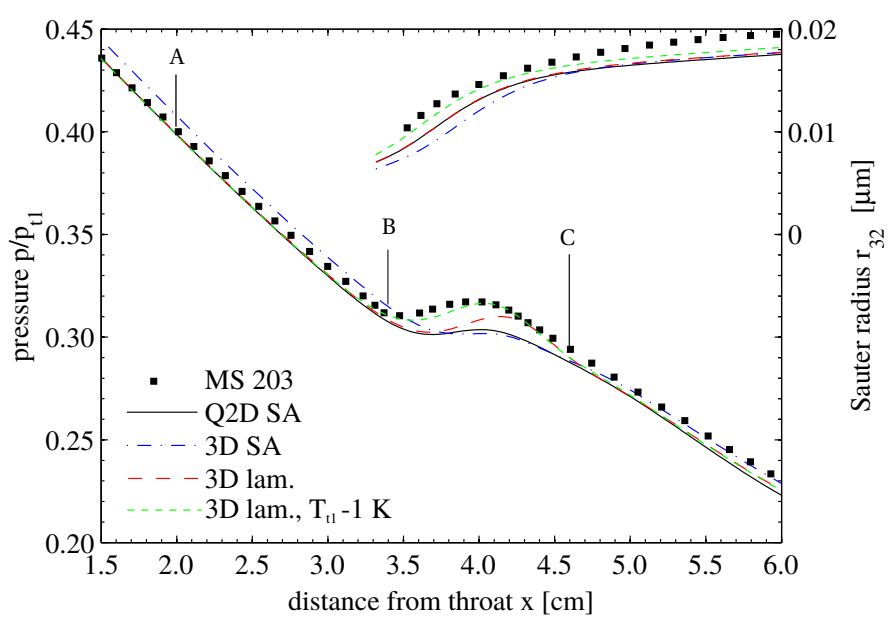

FIGURE 2. Pressure and droplet size along the centreline for the Moses and Stein nozzle case 203

case considered (case 203) has a total inlet pressure of $p_{\mathrm{t} 1}$ $=35.87 \mathrm{kPa}$ and a total inlet temperature of $T_{\mathrm{t} 1}=368.3 \mathrm{~K}$. The measured pressure distribution and droplet sizes have been taken from Ref. [1] because the original publication [8] does not contain the full data set.

Typical quasi two-dimensional (Q2D) fully-turbulent calculations are shown in Fig. 2 together with 3D laminar and 3D turbulent results. From the pressure distributions it can be seen that all three models predict condensation too far downstream. Aside from the previously mentioned uncertainties in nucleation and droplet growth, the accuracy of the experiments should also be considered. A decrease in inlet temperature of just $1 \mathrm{~K}$ would bring the location of the pressure plateau and the droplet size measurement in line with the numerical predictions, although Moses and Stein estimated that the measurement error is only $\pm \sim 0.5 \mathrm{~K}$. Unfortunately a definitive explanation of the discrepancies between calculation and experiment cannot be given; the difficulty is that there are so many possible causes relating to both the modelling and the measurements.

In the context of the present focus on boundary layer effects, the predicted pressure distributions have small but notable differences. Immediately downstream of the throat (up to the point labelled A) the Q2D and laminar 3D results agree well with experiment, but turbulent 3D pressures are significantly overpredicted. It should be recalled that the steam is dry in this region and so the uncertainties associated with condensation modelling are absent. The most likely cause of the over-predicted pressures is therefore incorrect calculation of the boundary layer growth.

An appreciation of boundary layer thickness can be obtained from Fig. 3 which shows computed total pressure contours on the cross sectional surface at the throat. The lines and numbers within the plots give the boundary layer thicknesses (based on $u_{99}$ ) for each calculation. Boundary layers on the curved and 


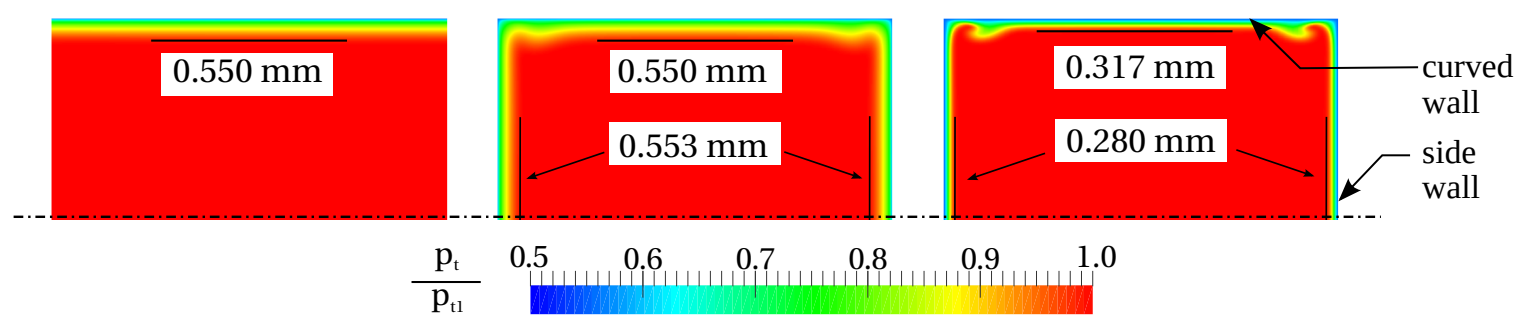

FIGURE 3. Boundary layer thickness for the Moses and Stein nozzle, case $203, p_{\mathrm{t} 1}=35.874 \mathrm{kPa}, T_{\mathrm{t} 1}=368.3 \mathrm{~K}$

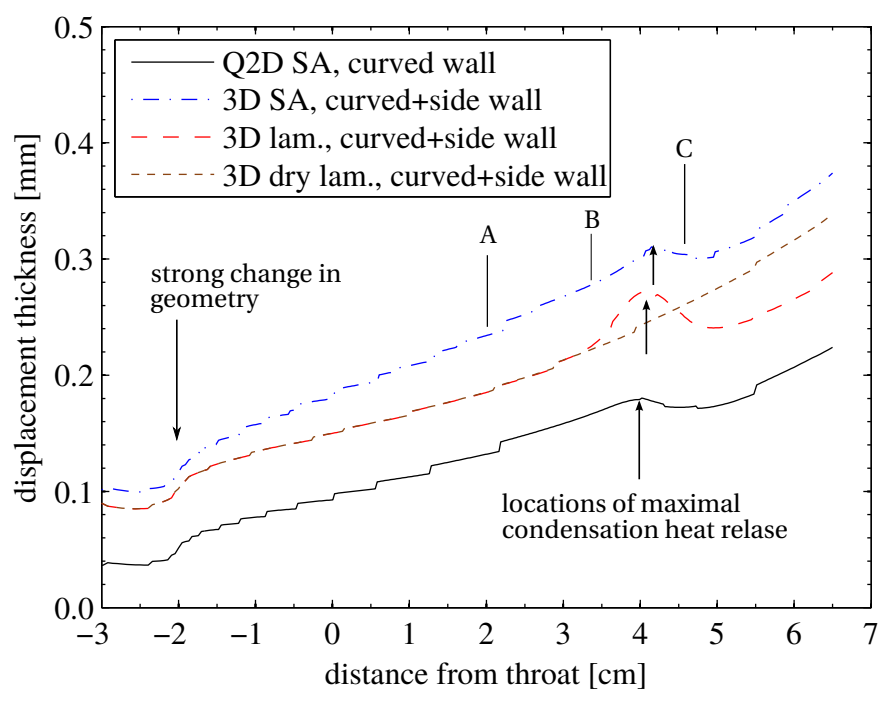

FIGURE 4. Displacement thickness along the nozzle for case 203

side walls are very similar, but thicknesses for the turbulent cases are almost twice those of the laminar result. Evidence of minor secondary flow features is also apparent in the corner regions for the 3D calculations, particularly for the laminar case.

Assuming 1D flow, the pressure distribution within the dry region is really determined by the ratio of the effective flow area to that at the throat. The influence of boundary layer growth is thus best understood in terms of the displacement thickness variations which are shown in Fig. 4. A sudden increase in thickness occurs around $x=-2 \mathrm{~cm}$ due to the transition between the large and the small wall radius (see Fig. 1), but beyond this the boundary layers grow steadily up to point B. Note that the sum of the side and curved wall thicknesses have been plotted, hence the 3D turbulent result is roughly twice that of the 2D case.

The displacement thickness for the 3D laminar and the 3D turbulent cases are similar in the converging section of the nozzle but the growth in thickness is slower downstream for the laminar calculation. This means that the differences between the 3D laminar and Q2D turbulent calculations become smaller and, although the mass flow rates are slightly different, the pressure distributions are almost identical. This trend ends when spontaneous condensation occurs, near point $\mathrm{B}$. At the position of maximal condensation heat release the boundary layers undergo local changes. Although the increase in wetness fraction (not shown in the paper) and thus the condensation heat release is very similar between the 3D laminar and the Q2D turbulent calculation, the effect on the boundary layer is much more pronounced for the laminar case. The displacement thickness for the 3D laminar calculation rises which leads to a different shape of the condensation pressure plateau compared to the turbulent calculations. Ultimately, this means that the condensation pressure rise not only depends on the condensation heat release but also reacts sensitively on the type of boundary layer present. Careful consideration of this matter is therefore required if multi-dimensional CFD techniques are to be used to calibrate wet steam models.

It is worth mentioning that a 1D model can overcome the uncertainties associated with modelling the boundary layers, if an effective flow area can be obtained from pressure measurements in a dry expansion. In Fig. 4 the displacement thickness of a dry 3D laminar calculation is given. After spontaneous condensation has occurred a difference in the displacement thickness between the wet and the dry calculation will exist and this would change the outlet pressure of a 1D calculation. However, the influence on the wetness fraction is only slight and the error in the droplet size will be below $2 \%$. (Despite the condensation-induced pressure rise the displacement thickness is slightly reduced after spontaneous condensation has occurred. This effect was also observed by Schnerr [32] and is explicable in terms of mixture density changes.)

At point $\mathrm{C}$ in Figs. 2 and Fig 4 the flow has almost returned to equilibrium and beyond this point the agreement with experiment is best using the 3D turbulent result. Although the evidence is relatively slim, these results together suggest that transition from a laminar to turbulent boundary layer may be occurring somewhere between points $\mathrm{A}$ and $\mathrm{C}$. That this is the case is supported by relatively high Reynolds numbers. The axiallength-based Reynolds number at the throat is $\operatorname{Re}_{x}=3 \times 10^{5}$ and well within the transition region. The strongly favourable pressure gradient within nozzles would tend to stabilise the laminar boundary layer, but transition may nonetheless be triggered 


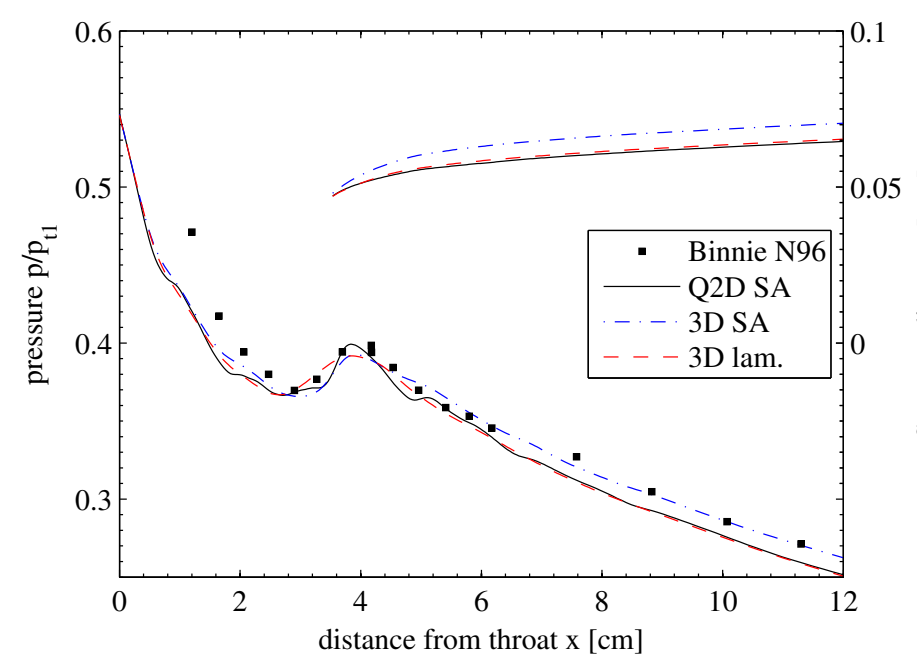

FIGURE 5. Binnie nozzle [10], N96, $p_{\mathrm{t} 1}=66.2 \mathrm{kPa}, T_{\mathrm{t} 1}=381.51 \mathrm{~K}$

by the condensation-induced pressure rise, just downstream of point $\mathrm{B}$.

\section{BINNIE NOZZLE}

Binnie et al. $[10,33]$ made pressure measurements along a less narrow nozzle with a throat height of $19.06 \mathrm{~mm}$ and a constant depth of $22.2 \mathrm{~mm}$. Measured area ratios are given in Ref. [10] and these have been used to obtain the geometry for the present calculations. The expansion rate is about $10000 \mathrm{~s}^{-1}$ near the throat. The converging part of the nozzle is shaped by cylindrical arcs which blend with a straight section downstream diverging at $2^{\circ}$. The change in curvature generates alternate regions of rapid and gradual expansion that show up as pressure undulations along the nozzle centreline. These are particularly evident in the measured dry expansion pressure distribution for this nozzle presented in Ref. [33,34].

Fig. 5 compares computed and measured pressure distributions for the Binnie nozzle under condensing flow conditions. The pressure measurement has been carried out in the centre of the side wall. The above-mentioned pressure undulations are evident in the Q2D calculations, but tend to get smeared by boundary layer growth in the other cases. Boundary layer growth on the side wall generates weak compression waves which may also help to weaken the expansion waves emanating from the curvature discontinuity [35].

None of the calculations agrees with the measured pressure upstream of the condensation zone, but it is notable that the calculations of Hill et al. [20] also show the same discrepancy. There is some uncertainty in the geometric data available from the references, but differences would need to be very significant to obtain the measured pressure at the most upstream location

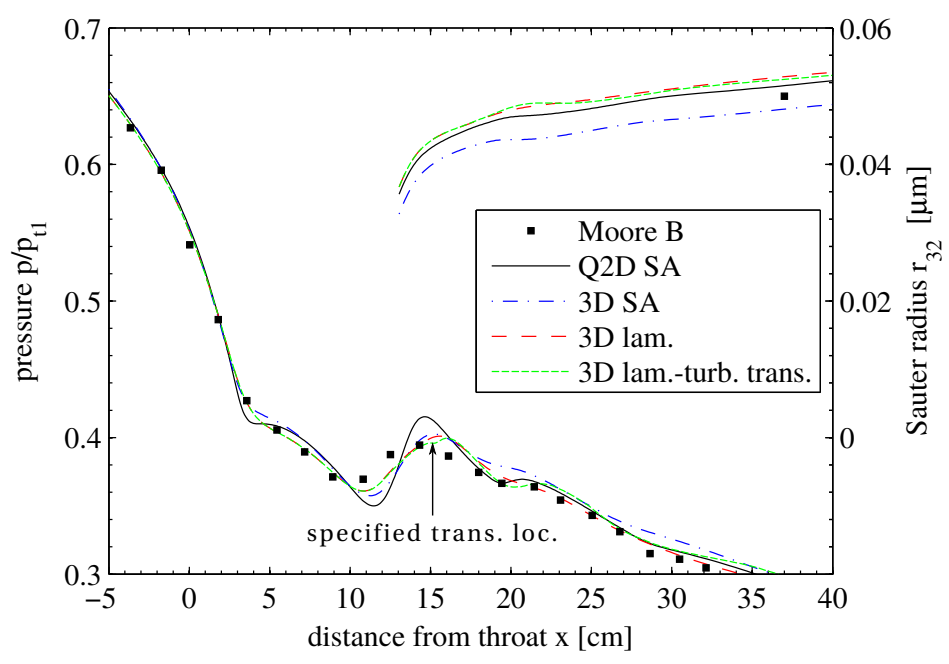

FIGURE 6. Moore nozzle B [9], $p_{\mathrm{t} 1}=25.0 \mathrm{kPa}, T_{\mathrm{t} 1}=358.1 \mathrm{~K}$

and it may be that this measurement is in error. Unlike the Moses and Stein case, differences between the turbulent, laminar and Q2D results are not significant in this region due to the larger nozzle dimensions.

Downstream of the condensation zone the laminar and Q2D calculations underpredict the pressure, especially towards the end of the nozzle. Again it would seem plausible that transition occurs within or near the condensation zone, and this is supported by Reynolds number considerations. (The distance-based Reynolds number is $5 \times 10^{5}$ at the throat and increases to $8 \times 10^{5}$ at $x=4 \mathrm{~cm}$, suggesting that transition is likely.)

\section{MOORE NOZZLE B}

A commonly used test case is Moore et al.'s nozzle B [9]. This nozzle has a modest expansion rate of about $2300 \mathrm{~s}^{-1}$ since it is much longer than the previous nozzles. The throat height is $100 \mathrm{~mm}$ and the constant depth of the nozzle is $152 \mathrm{~mm}$. The geometry and grid are available in the IWSMP collaboration [30]. The geometry is similar to the Binnie nozzle (blended circular arc and straight line section) giving rise to similar pressure undulations. However, in comparison to the Binnie nozzle these are much more pronounced and clearly visible in the centreline (side wall) pressure measurements shown in Fig. 6.

As with the Binnie nozzle, there is very little difference between the various calculations in the rapidly accelerating upstream section $(x<2 \mathrm{~cm})$ due to the large nozzle dimensions. Discrepancies arise further downstream as a consequence of the geometry-induced strong pressure undulations. These are again smoothed out by the side wall boundary layers in the 3D calculations and it is notable that the agreement with the experimental pressure distribution in the zone of spontaneous condensation 
is much better with the 3D calculations. Differences in the expansion rate at the Wilson point arising from the various boundary layer assumptions lead to slight differences in the predicted droplet size, but these are all within $10 \%$ of the measured value and thus within the range of experimental uncertainty.

Consideration of the Reynolds number $\left(\operatorname{Re}_{x} \simeq 4 \times 10^{5}\right.$ at the throat) would again suggest that transition to turbulence is likely to occur, but it is noteworthy that the laminar results are much closer to the measurements throughout the nozzle. A possible explanation for this is that because the pressure undulations stem from expansion waves emanating from the curvature discontinuity, they are sensitive to the boundary layer modelling. The interaction of the expansion waves with the opposite walls and especially the generation of downstream compression waves are strongly influenced by the state of the boundary layers. (In this respect, differences between the 3D turbulent and laminar results are evident at the first undulation but also further downstream.) To test this hypothesis, 3D calculations were undertaken with transition set at the local pressure maximum where spontaneous condensation ends. It can be seen in Fig. 6 that the transition calculation gives an agreement with the experiments that is at least as good as the laminar result. This confirms that for nozzles with strong two-dimensional structures the state of the boundary layers and also the transition process can significantly influence the shape of the pressure undulations.

\section{GYARMATHY'S LOW PRESSURE NOZZLES}

The previous results show that assumptions about the boundary layer can have a notable impact on computed nozzle pressure distributions, which in turn have implications for the validation of condensing flow routines. This is especially so for small nozzles for which the size of the boundary layer is a significant fraction of the nozzle width, but it is also true for the larger nozzles if they exhibit strong two-dimensional effects. In any case, correct prediction of the viscous effects is an important aspect of flow modelling and a more detailed study of the boundary layers is warranted. Unfortunately, most of the published data for condensation in nozzles contain no information on this important topic. The only exception, to the authors' knowledge, is the study by Gyarmathy and Meyer [16] which includes some boundary layer profile data, albeit only for dry expansions.

Gyarmathy and Meyer measured pressure distributions for small, medium and large nozzles with the aim of determining the influence of expansion rate on the Wilson point pressure. Boundary layer measurements were made in order to establish the real expansion on the $h-s$ chart, rather than rely on assumptions of isentropic flow or otherwise. Unfortunately, no direct measurements of droplet sizes were made for the wet steam cases, so only the dry expansions are considered here. (The boundary layer conditions and pressure measurements of the wet steam experiments can be found in Ref. [16]). The boundary layer data are
TABLE 1. Geometrical data for the Gyarmathy nozzles

\begin{tabular}{lcc}
\hline Variable & $\begin{array}{c}\text { small } \\
\text { nozzle }\end{array}$ & $\begin{array}{c}\text { medium } \\
\text { nozzle }\end{array}$ \\
\hline design expansion rate $\dot{P}\left[\mathrm{~s}^{-1}\right]$ & 10000 & 3333 \\
base length $l_{0}[\mathrm{~mm}]$ & 115.3 & 346.0 \\
channel depth $b[\mathrm{~mm}]$ & 14.0 & 24.0 \\
height at the throat $h_{\mathrm{T}}[\mathrm{mm}]$ & 10.0 & 17.1 \\
height at the end of the nozzle $h_{\mathrm{E}}[\mathrm{mm}]$ & 20.4 & 34.9 \\
\hline horizontal inlet length $l_{\text {in }}[\mathrm{mm}]$ & 10 & 30 \\
axial coordinate $x_{\mathrm{A}}[\mathrm{mm}]$ & -70.0 & -140 \\
height $h_{\mathrm{A}}[\mathrm{mm}]$ & 33.52 & 54.34 \\
\hline
\end{tabular}

exploited here as a means of investigating the suitability of different turbulence models in the context of strongly accelerating nozzle flows.

\section{Geometry}

The nozzle geometry of the small and the medium nozzle are shown in Fig. 7 and also given in the appendix. The large nozzle were of a similar construction, but is not considered here because it is suspected that some of the results were corrupted by leakage effects [36, p. 38]. Most geometric details are available in Ref. [16] and nozzle shapes derived from this publication. There is some uncertainty regarding the location of the parallel inlet and the geometry of the converging section for which it is only known that it is of parabolic form. However, in Ref. [36] Gyarmathy and Meyer provide the 'theoretical' pressure ratio variation, obtained on the basis of a perfect gas, isentropic expansion. This can be used to derive a major part of the inlet geometry using standard 1D compressible flow theory. Unfortunately there is no information regarding the blend point between the parallel and parabolic sections, thus $l_{\text {in }}$ and $h_{\mathrm{A}}$ (see Fig. 7) are more or less arbitrarily chosen.

The diverging part of the nozzles is designed to provide a constant expansion rate. To achieve this Gyarmathy and Meyer derived a set of governing equations based on 1D isentropic theory. The target expansion rate, length of the nozzle and the throat height are first specified and the governing differential equation then has to be integrated numerically. This process is described in detail in the appendix, and the main geometric details for the two nozzles are summarised in Tab. 1. 

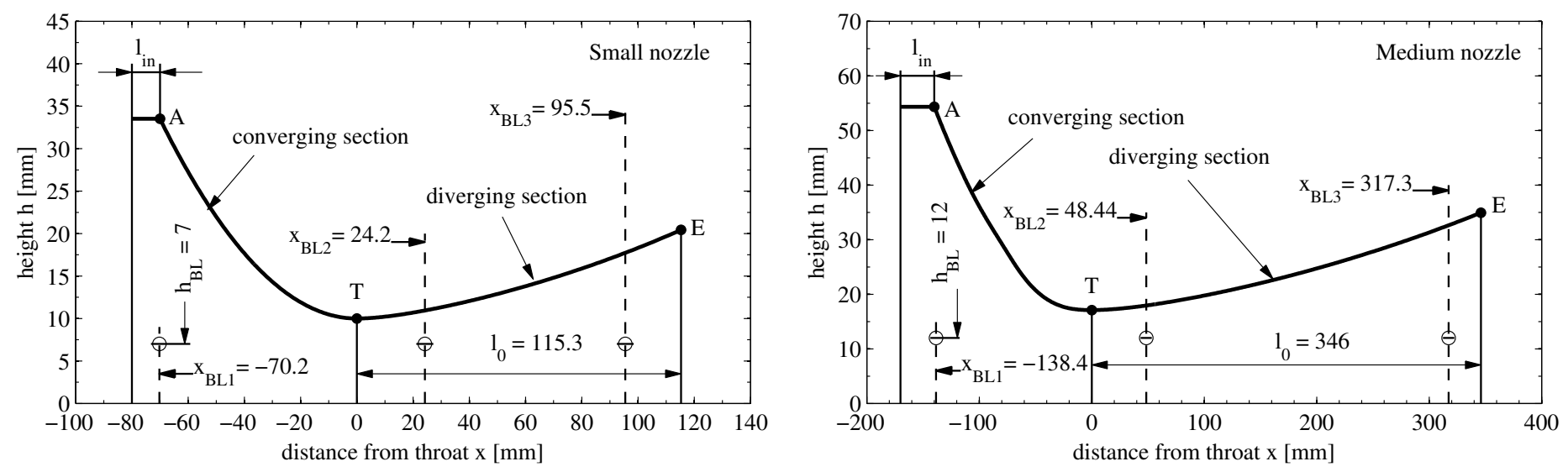

FIGURE 7. Geometry of the small and medium Gyarmathy nozzles

\section{Experimental setup}

For each test, upstream stagnation conditions were recorded with a Pitot probe and thermocouple, with stated experimental uncertainties of $\pm 0.5 \%$ in pressure and $\pm 1 \mathrm{~K}$ respectively. Static pressure along the bottom wall was obtained with a sliding bar containing 5 tappings. Boundary layer measurements were made on the plane side walls using both a circular and flattened Pitot probes. The stated accuracy of the traversing system is 0.1 $\mathrm{mm}$. For measurements in the supersonic region, the standard bow shock correction was applied, namely

$$
\frac{p_{\mathrm{t}}}{p_{\mathrm{Pit}}}=\left[1+\frac{2}{\gamma+1}\left(\mathrm{Ma}^{2}-1\right)\right]^{\frac{1}{\gamma-1}}\left[1-\frac{2 \gamma}{\gamma+1}\left(1-\frac{1}{\mathrm{Ma}^{2}}\right)\right]^{\frac{\gamma}{\gamma-1}}
$$

where

$$
\mathrm{Ma}=\sqrt{\frac{2}{\gamma-1}\left[\left(\frac{p_{\mathrm{t}}}{p}\right)^{\frac{\gamma-1}{\gamma}}-1\right]} .
$$

In the following figures symbols are used to represent the experimental data. It has to be mentioned that the position of the symbols don't reflect a real measurement position as Gyarmathy and Meyer [16] have shown their experimental data by lines.

The dry operating conditions and the measurements available from Ref. [16] are summarised in Tab. 2 for the small and medium nozzle respectively. Note, however, that boundary layers were measured for an inlet pressure of $110 \mathrm{kPa}$, whereas centreline pressure measurements are only available for a lower inlet pressure.
TABLE 2. Boundary conditions for the Gyarmathy nozzles

\begin{tabular}{ccc} 
Small nozzle & $p_{\mathrm{t} 1}[\mathrm{kPa}]$ & $T_{\mathrm{t} 1}[\mathrm{~K}]$ \\
\hline $\mathrm{P}$ & 50.0 & 453.0 \\
$\mathrm{BL}$ & 110.0 & 483.0 \\
\hline Medium nozzle & & \\
\hline $\mathrm{P}$ & 50.0 & 488.0 \\
$\mathrm{BL}$ & 110.0 & 483.0 \\
\hline
\end{tabular}

\section{Dry steam results}

In the following the numerical results are compared to the available experimental data for dry steam cases. The $y_{+}$-values of the grid are less than one requiring grid sizes of $400 \times 121 \times$ 121 for the small nozzle and $570 \times 141 \times 141$ for the medium nozzle. In Fig. 8 the pressure distributions along the wall for the small and medium nozzle are presented. For both nozzles the inlet total pressure is $50 \mathrm{kPa}$ while the total temperature varies.

Upstream of the throat hardly any difference between the 3D laminar and 3D turbulent calculations can be seen. This is consistent with previous findings as the throat area of the small Gyarmathy nozzle is larger than the Moses and Stein nozzle. For the 3D laminar simulations the pressures downstream of the throat are below the measurements which indicates that the laminar model underpredicts the measured boundary layer thickness. The agreement with the measurements is considerably better for the turbulent calculations. The Reynolds numbers at the throat are $\operatorname{Re}_{\mathrm{x}}=3 \times 10^{5}$ for the small and $8 \times 10^{5}$ for the medium nozzle. As for the previously investigated nozzle cases boundary layer transition can be expected at or after the throat and might be a reason for the remaining small discrepancies. 


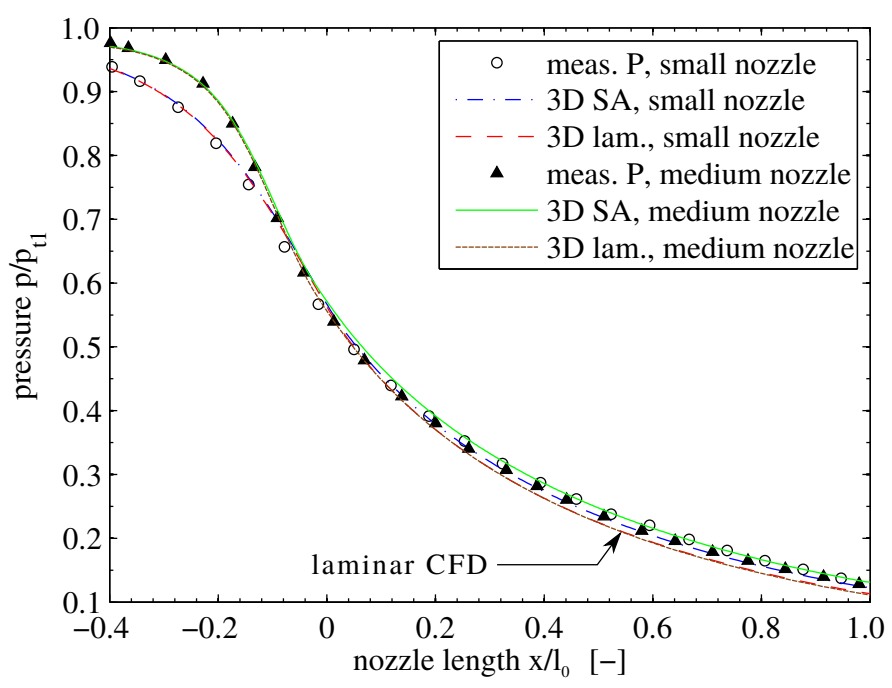

FIGURE 8. Pressure distributions for dry cases with $p_{\mathrm{t} 1}=50 \mathrm{kPa}$

Gyarmathy and Meyer measured the boundary layer at three different axial positions ( $x_{\mathrm{BL} 1}, x_{\mathrm{BL} 2}, x_{\mathrm{BL} 3}$, see Fig. 7). In Fig. 9 these measurements in the small (left) and in the medium nozzle (right) are compared to the numerical results. This comparison includes results from the commercial flow solver Ansys CFX using the same grid and the same turbulence model. Little can be said about the first measurement, at position I, because the boundary layer is still very small. For the small nozzle the profile at location II downstream of the throat is well-predicted by Steamblock whereas CFX slightly underestimates the boundary layer thickness. At position III the CFX result gives a better agreement with the experimental data and Steamblock overestimates the boundary layer thickness. Interestingly, the laminar results of CFX and Steamblock are almost identical but it seems that there is a difference in the implementation of the SpalartAllmaras model. For the medium nozzle the same comparison is given on the right hand side of Fig. 9. The calculated boundary profiles of Steamblock and CFX are more similar than in the small nozzle case but the experimental data show a noticeably broader boundary layer at positions II and III.

The choice of turbulence model is often a critical decision because it can affect results considerably. For this reason CFX has been used to investigate the influence of standard turbulence models and the results are presented in Fig 10. In the near wall region the SST model is equal to the k-omega model and thus similar result are obtained for the inner boundary layer. However, in the outer region the pure k-omega model tends to achieve better agreement with the measurements. This is especially true for the medium nozzle, where both the SST and the SA model underpredict the boundary layer growth.

In the measurements for the small and especially for the medium nozzle a total pressure loss in the core flow of the noz- zles is observed. A possible explanation for this is early heterogeneous condensation which has not been modelled numerically. However, the formation of droplets should have attracted attention because Gyarmathy and Meyer were able to observe the flow through a window during at least some experiments. In a dry flow, a loss in total pressure can only occur due to viscous effects or due to shock waves, and no signs for the latter are noticeable in the experimental data. Gyarmathy and Meyer suggest that a high level of free stream turbulence causes the high core flow losses. Calculations with a reasonably high level of free stream turbulence have shown that this has almost no effect on the boundary layer profiles. This is because free stream turbulence decays very quickly in highly accelerated nozzle flows. Another source for viscous losses could be a flow separation which may occur due to the experimental instrumentation within the nozzle. However, without more detailed information about the geometry and how the measurements have been carried out it is difficult to find a reliable explanation for the high core flow losses.

All of the turbulence models are able to predict the boundary layers with modest accuracy but non of them does significantly better than the others. The cases with boundary layer measurements have inlet pressures of $100 \mathrm{kPa}$ with distance-based Reynolds numbers at the throat of $\operatorname{Re}_{\mathrm{x}}=8 \times 10^{5}$ (small nozzle) and $2 \times 10^{6}$ (medium nozzle). As with the previously discussed cases, transition will be occurring somewhere upstream and the success of turbulence models cannot be fully assessed without consideration of this process.

\section{CONCLUSIONS}

The present paper discusses the influence of boundary layers on the steam flow in nozzles with a particular focus on the implications for the validation and calibration of condensation models. It may be concluded that the traditional one-dimensional method of interpreting the effective nozzle geometry from dry expansion pressure distributions is likely to be reliable, provided the nozzle is free from strong two-dimensional pressure undulations. Changes in boundary layer blockage due to the condensation pressure rise then only affect the pressure distribution downstream of nucleation and the likely impact on the predicted final droplet size is only a few percent.

Several of the well-known nozzle test cases do nonetheless exhibit strong two-dimensional effects and modern CFD methods have the potential to capture these accurately. Care must be taken however in employing such methods to validate wet steam models, especially in regard to the growth of boundary layers. All boundary layers (profiled walls and side walls) must obviously be accounted for, but the state of these boundary layers should also be considered. The results presented here are all consistent with a laminar layer being sustained for a substantial part of the nozzle and transition occurring within or slightly downstream of the throat region. In particular, the frequently- 

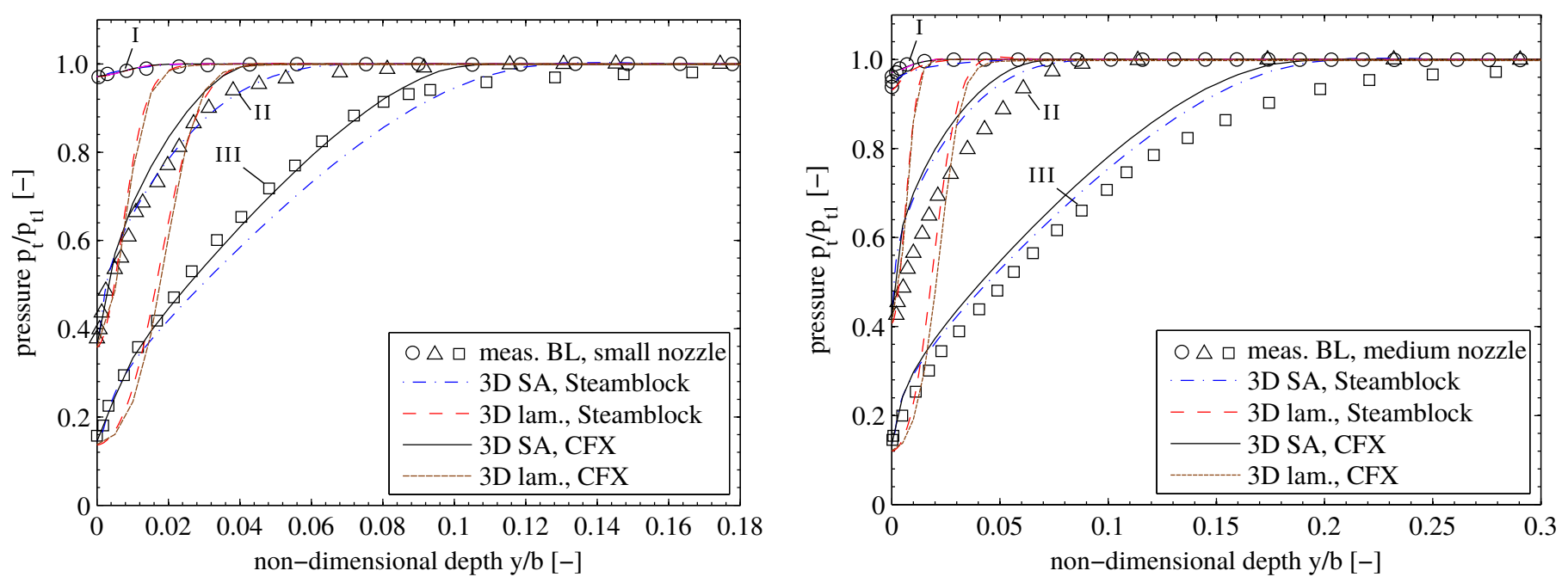

FIGURE 9. Comparison of laminar and turbulent calculations, left: small nozzle, right: medium nozzle
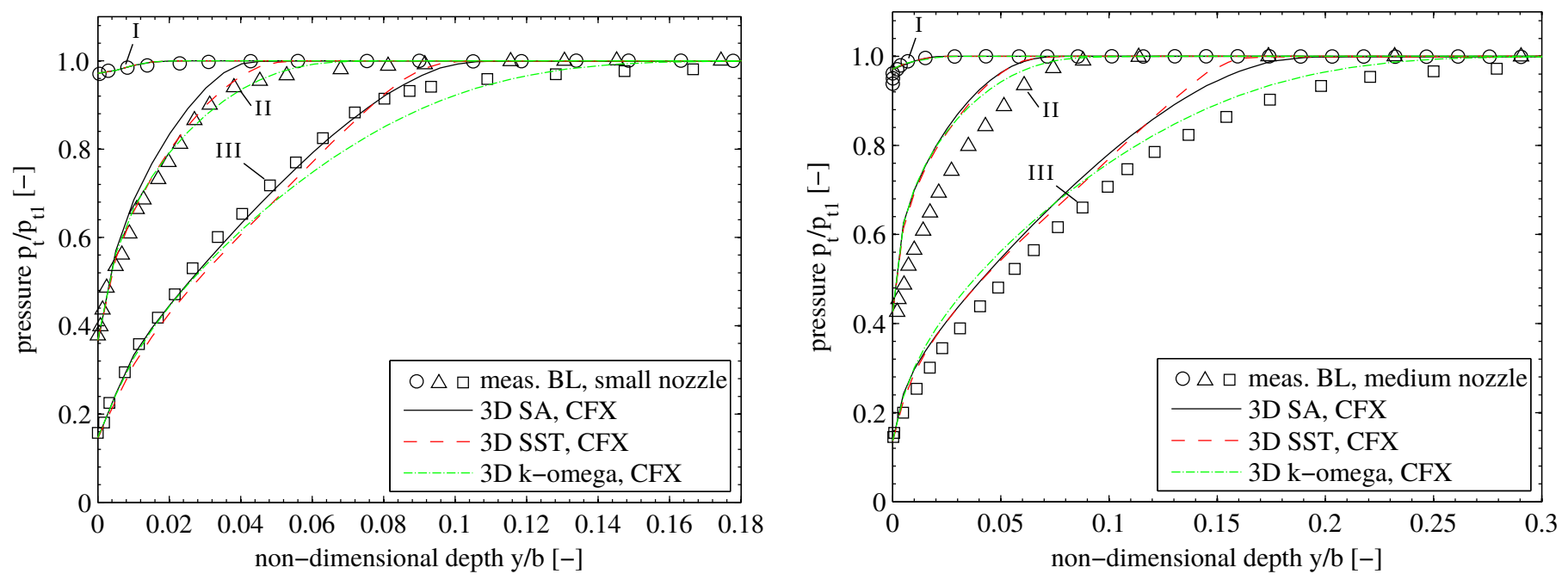

FIGURE 10. Comparison of different turbulence models, left: small nozzle, right: medium nozzle

used tests of Moses and Stein and Moore et al.are investigated. These are sensitive to boundary layer assumptions due to the small throat area (in the first case) and discontinuity in profile curvature (in the second), and pressure distributions cannot be accurately predicted, even in dry regions of the flow, unless transition is assumed to occur beyond the throat.

The nozzle tests of Gyarmathy and Meyer are the only ones to include detailed boundary layer profile measurements, albeit only for dry expansions. These confirm that boundary layers are turbulent downstream of the throat but the data cannot provide any information on where transition occurs. Dry calculations conducted with different turbulence models (Spalart-Allmaras, SST and k-omega) show that all of these give satisfactory prediction of the blockage effect (as shown by centreline pressure distributions) and modest agreement with the measured velocity profiles. None of the turbulence models may be deemed superior in terms of the level of agreement obtained.

Finally it is worth noting that, despite the many test cases reported in the literature, not one of the condensing nozzle flow experiments provides a comprehensive set of data. Such an experiment would be extremely valuable to the wet steam community and would comprise: a well designed nozzle, free from two-dimensional pressure undulations; measurements of centreline pressure distribution in both wet and dry flow; light extinction measurements over a range of wavelengths and at different locations to obtain reliable droplet size data; velocity profile measurements at various axial locations, including upstream of the throat. 


\section{ACKNOWLEDGEMENT}

The authors are grateful to Prof. John Young for his valuable comments about wet steam flow modelling. The authors also acknowledge the helpful discussions with Prof. Holger Babinsky. The presented work is part of the project "Future Conventional Power Consortium" and the authors are thankful to EPSRC for funding this research.

\section{REFERENCES}

[1] Young, J. B., 1982. "The spontaneous condensation of steam in supersonic nozzles". PhysicoChemical Hydrodynamics, 3(1), pp. 57-82.

[2] Young, J. B., 1992. "Two-dimensional, nonequilibrium, wet-steam calculations for nozzles and turbine cascades". Trans. ASME, J. Turbomach., 114(3), July, pp. 569-579.

[3] S̆ St' astný, M., S̆ejna, M., and Jonas, O., 1997. "Modeling the Flow with Condensation and Chemical Impurity in Steam Turbine Cascade". In Proc. 2nd Europ. Conf. on Turbomachinery, pp. 81-88.

[4] Wróblewski, W., Dykas, S., Gardzilewicz, A., and Kolovratnik, M., 2009. "Numerical and experimental investigations of steam condensation in LP part of a large power turbine". Trans. ASME, J. Fluids Eng., 131(4), pp. 1-11.

[5] Chandler, K. D., Melas, M., and Jorge, T., 2015. "A study of spontaneous condensation in an LP test turbine". In ASME Conf. Proc. Turbo Expo 2015, "15-19" June, Montreal, Canada.

[6] Starzmann, J., Casey, M., Mayer, J. F., and Sieverding, F., 2014. "Wetness loss prediction for a low pressure steam turbine using cfd". Proc. IMechE, Part A: J. Power and Energy, 228(2), pp. 216-231.

[7] Zhu, X., Yuan, X., Lin, Z., Shibukawa, N., Tsukuda, T., Niizeki, Y., and Tanuma, T., 2013. "An upwind eulerianeulerian model for non-equilibrium condensation steam turbines". In Proceedings of ASME Turbo Expo 2013: Power for Land, Sea and Air.

[8] Moses, C. A., and Stein, G. D., 1978. "On the growth of steam droplets formed in a laval nozzle using both static pressure and light scattering measurements". Trans. ASME, J. Fluids Eng., 100, pp. 311-322.

[9] Moore, M. J., Walters, P. T., Crane, R. I., and Davidson, B. J., 1973. "Predicting the fog-drop size in wet-steam turbines". In Proc. Wet Steam 4, IMechE.

[10] Binnie, A. M., and Green, J. R., 1942. "An electrical detector of condensation in high-velocity steam". Proc. R. Soc. Lond. A(181), pp. 134-153.

[11] Barschdorff, D., 1971. "Verlauf der Zustandsgrößen und gasdynamische Zusammenhänge bei der spontanen Kon- densation reinen Wasserdampfes in Lavaldüsen". Forsch. Ingenieurwes., 37(5), pp. 146-157.

[12] Oswatitsch, K., 1942. "Kondensationserscheinungen in Überschalldüsen". Z. Angew. Math. Mech., 22(1), Feb., pp. 1-14.

[13] White, A. J., and Young, J. B., 1993. "Time-marching method for the prediction of two-dimensional, unsteady flows of condensing steam". AIAA J. Propulsion and Power, 9(4), July-Aug., pp. 579-587.

[14] Simpson, D. A., and White, A. J., 2005. "Viscous and unsteady flow calculations of condensing steam in nozzles". Int. J. Heat and Fluid Flow, 26, pp. 71-79.

[15] Wróblewski, W., Dykas, S., and Gepert, A., 2009. "Steam condensing flow modeling in turbine channels". Int. J. Multiphase Flow, 35(4), Mar., pp. 498-506.

[16] Gyarmathy, G., and Meyer, H., 1965. "Spontane Kondensation Teil 2: Einfluss der Entspannungsschnelligkeit auf die Nebelbildung in übersättigtem Dampf'. VDIForschungsheft 508, VDI-Verlag.

[17] Denton, J. D., 1992. "The calculation of three-dimensional viscous flow through multistage turbomachines". Trans. ASME, J. Turbomach., 114, pp. 18-26.

[18] Pullan, G., and Denton, J. D., 2003. "Numerical simulations of vortex-turbine blade interaction". In 5th European Conference on Turbomachinery - Fluid Dynamics and Thermodynamics, pp. 1049-1059.

[19] Chandler, K. D., White, A. J., and Young, J. B., 2014. "Non-equilibrium wet-steam calculations of unsteady lowpressure turbine flows". Proc. IMechE, Part A: J. Power and Energy, 228(2), pp. 143-152.

[20] Hill, P. G., Miyagawa, K., and Denton, J. D., 2000. "Fast and accurate inclusion of steam properties in two- and three-dimensional steam turbine flow calculations". Proc. Inst. Mech. Eng. C, J. Mech. Eng. Sci., 214(7), pp. 903919.

[21] The International Association for the Properties of Water and Steam, 2007. Revised Release on the IAPWS Industrial Formulation 1997 for Thermodynamic Properties of Water and Steam. Internet resource.

[22] The International Association for the Properties of Water and Steam, 2008. Release on the IAPWS Formulation 2008 for Viscosity of Ordinary Water Substance. Internet resource.

[23] The International Association for the Properties of Water and Steam, 2011. Release on the IAPWS Formulation 2011 for the Thermal Conductivity of Ordinary Water Substance. Internet resource.

[24] The International Association for the Properties of Water and Steam, 2014. Revised Release on Surface Tension of 
Ordinary Water Substance. Internet resource.

[25] Bakhtar, F., Young, J. B., White, A. J., and Simpson, D. A., 2005. "Classical nucleation theory and its application to condensing steam flow calculations". Proc. Inst. Mech. Eng. C, J. Mech. Eng. Sci., 219(12), pp. 1315-1333.

[26] Hill, P. G., 1966. "Condensation of water vapour during supersonic expansion in nozzles". J. Fluid Mech., 25, pp. 593-620.

[27] White, A. J., and Hounslow, M. J., 1999. "Modelling droplet size distributions in polydispersed wet-steam flows". Int. J. Heat and Mass Transfer, 43(11), Sept., pp. 1873-1884.

[28] White, A. J., 2003. "A comparison of modelling methods for polydispersed wet-steam flow". Int. J. Numer. Meth. Engrg., 57, pp. 819-834.

[29] Hughes, F. R., Starzmann, J., White, A. J., and Young, J. B., 2015. "A comparision of modeling techniques for polydispersed droplet spectra in steam turbines". Trans. ASME, J. Eng. Gas Turb. Power, 138(4), Oct.

[30] International Wet Steam Modelling Project, 2015. University Cambridge, http://wet-steam-project.eng.cam.ac.uk.

[31] ANSYS, I., 2015. ANSYS CFX-Solver Theory Guide, Release 16.2. ANSYS, Inc.

[32] Schnerr, G. H., Bohning, R., Breitling, T., and Jantzen, H. A., 1992. "Compressible turbulent boundary layers with heat addition by homogeneous condensation". AIAA J., 30(5), May, pp. 1284-1289.

[33] Binnie, A. M., and Woods, M. W., 1938. "The pressure distribution in a convergent-divergent steam nozzle". Proc. Instn. Mech. Eng., 138, pp. 229-266.

[34] Binnie, A. M., 1950. "Notes on gas flow through a nozzle". Mathematical Proc. Cambridge Philosopical Society, 46, July, pp. 492-499.

[35] Babinsky, H., 2015. Boundary layers in nozzle flows. Private Communication.

[36] Gyarmathy, G., 1976. "Condensation in flowing steam". In Two Phase Steam Flow in Turbines and Separators, M. J. Moore and C. H. Sieverding, eds. Hemisphere Publishing Corporation.

\section{Appendix}

This section summarises the method used be Gyarmathy and Meyer [16] to design the diverging section of nozzles with a constant expansion rate. The expansion rate is defined as,

$$
\dot{P}=-\frac{1}{p} \frac{\mathrm{d} p}{\mathrm{~d} t}
$$

and the time derivative $\mathrm{d} t$ can be expressed as,

$$
\mathrm{d} t=\frac{\mathrm{d} x}{c}
$$

Both equations can be combined together to give,

$$
-\frac{c}{p} \frac{\mathrm{d} p}{\mathrm{~d} x}=\dot{P}
$$

The velocity $c$ is still unknown but for one-dimensional, isentropic and ideal gas flows the following method can be used. The caloric equation of state $\mathrm{d} h=c_{\mathrm{p}} \mathrm{d} T$ and the isentropic relation,

$$
\frac{T}{T_{\mathrm{t}}}=\left(\frac{p}{p_{\mathrm{t}}}\right)^{\frac{\gamma-1}{\gamma}}
$$

can be substituted into the energy equation $h_{\mathrm{t}}=h+1 / 2 c^{2}$. This leads to,

$$
\frac{c^{2}}{2 h_{\mathrm{t}}}=1-\left(\frac{p}{p_{\mathrm{t}}}\right)^{\frac{\gamma-1}{\gamma}}
$$

Eq. (10) when applied to eq. (8) gives the differential equation,

$$
-\frac{p}{p_{\mathrm{t}}} \sqrt[-1]{1-\left(\frac{p}{p_{\mathrm{t}}}\right)^{\frac{\gamma-1}{\gamma}}} \mathrm{d}\left(p / p_{\mathrm{t}}\right)=\frac{\dot{P}}{\sqrt{2 h_{\mathrm{t}}}} \mathrm{d} x
$$

In [36] an analytical solution is given but Gyarmathy and Meyer [16] used a numerical integration method to solve eq. (11). The boundary condition at the throat $(x=0)$ is known because sonic conditions are reached there. By means of common gas dynamic relationships the pressure ratio at $\mathrm{Ma}^{*}=1$ equates to,

$$
\frac{p}{p_{\mathrm{t}}}=\frac{p^{*}}{p_{\mathrm{t}}}=\frac{2}{\gamma+1}^{\frac{\gamma}{\gamma-1}}
$$

For the lower integration limit the pressure ratio $p / p_{\mathrm{t}}$ is set to 0.1 which also provides the nozzle base length $l_{0}$ (Tab. 1 ). Note that for the isentropic exponent $\gamma=1.32$ and for the stagnation enthalpy $h_{\mathrm{t}}=813 \mathrm{KJ} / \mathrm{kg}$ is used.

Knowing the pressure ratio that result in a specified expansion rate the area ratio of the nozzle can be calculated. Using the density ratio,

$$
\frac{\rho}{\rho_{\mathrm{t}}}=\left(\frac{p}{p_{\mathrm{t}}}\right)^{\frac{1}{\gamma}}
$$


and eq. (10) as well as the continuity equation $(\dot{m}=A \rho c)$ the mass flow rate can be expressed by,

$$
\dot{m}=A \rho_{\mathrm{t}}\left(\frac{p}{p_{\mathrm{t}}}\right)^{\frac{1}{\gamma}} \sqrt{2 h_{\mathrm{t}}\left(1-\left(p / p_{\mathrm{t}}\right)^{\frac{\gamma-1}{\gamma}}\right)} .
$$

According to the flow conditions (eq. (12)) and the area $A^{*}$ at the throat the mass flow rate is also

$$
\dot{m}=A^{*} \rho_{\mathrm{t}}\left(\frac{2}{\gamma+1}\right)^{\frac{1}{\gamma-1}} \sqrt{2 h_{\mathrm{t}}(\gamma-1) /(\gamma+1)}
$$

Combining eq. (14) together with eq. (15) leads to,

$$
\frac{A}{A^{*}}=\left(\frac{2}{\gamma+1}\right) \sqrt{\frac{\gamma-1}{\gamma+1}}\left(\frac{p}{p_{\mathrm{t}}}\right)^{-\frac{1}{\gamma}} \sqrt{1-{\frac{p}{p_{\mathrm{t}}}}^{\frac{\gamma-1}{\gamma}}}
$$

which finally defines the nozzle geometry.

TABLE 3. Geometry of Gyarmathy's [16] small nozzle

\begin{tabular}{cccc}
\hline $\mathrm{x}[\mathrm{mm}]$ & $\mathrm{h}[\mathrm{mm}]$ & $\mathrm{x}[\mathrm{mm}]$ & $\mathrm{h}[\mathrm{mm}]$ \\
\hline-80.000 & 33.520 & -70.000 & 33.520 \\
-63.382 & 29.283 & -56.764 & 25.467 \\
-50.146 & 22.070 & -43.529 & 19.095 \\
-36.911 & 16.540 & -30.293 & 14.405 \\
-23.675 & 12.690 & -17.057 & 11.397 \\
-10.439 & 10.523 & -3.821 & 10.070 \\
0.000 & 10.000 & & \\
2.796 & 10.021 & 9.414 & 10.195 \\
16.032 & 10.483 & 22.650 & 10.848 \\
29.268 & 11.271 & 35.886 & 11.742 \\
42.504 & 12.256 & 49.121 & 12.809 \\
55.739 & 13.401 & 62.357 & 14.029 \\
68.975 & 14.693 & 75.593 & 15.395 \\
82.211 & 16.134 & 88.829 & 16.912 \\
95.446 & 17.729 & 102.064 & 18.587 \\
108.682 & 19.486 & 115.300 & 20.429 \\
(Spline interpolation has been used to generate the grid.) \\
\hline
\end{tabular}

TABLE 4. Geometry of Gyarmathy's [16] medium nozzle

\begin{tabular}{cccc}
\hline $\mathrm{x}[\mathrm{mm}]$ & $\mathrm{h}[\mathrm{mm}]$ & $\mathrm{x}[\mathrm{mm}]$ & $\mathrm{h}[\mathrm{mm}]$ \\
\hline-170.000 & 54.340 & -140.000 & 54.340 \\
-131.621 & 50.019 & -123.241 & 45.940 \\
-114.862 & 42.128 & -106.483 & 38.608 \\
-98.103 & 35.403 & -89.724 & 32.484 \\
-81.345 & 29.704 & -72.966 & 26.942 \\
-64.586 & 24.331 & -56.207 & 22.097 \\
-47.828 & 20.349 & -39.448 & 19.044 \\
-31.069 & 18.133 & -22.690 & 17.558 \\
-14.310 & 17.245 & -5.931 & 17.119 \\
0.000 & 17.100 & & \\
2.448 & 17.103 & 10.828 & 17.158 \\
19.207 & 17.268 & 27.586 & 17.420 \\
35.966 & 17.606 & 44.345 & 17.821 \\
52.724 & 18.061 & 61.103 & 18.322 \\
69.483 & 18.602 & 77.862 & 18.899 \\
86.241 & 19.213 & 94.621 & 19.541 \\
103.000 & 19.883 & 111.379 & 20.238 \\
119.759 & 20.606 & 128.138 & 20.986 \\
136.517 & 21.378 & 144.897 & 21.782 \\
153.276 & 22.198 & 161.655 & 22.624 \\
170.035 & 23.062 & 178.414 & 23.512 \\
186.793 & 23.972 & 195.172 & 24.444 \\
203.552 & 24.927 & 211.931 & 25.421 \\
220.310 & 25.926 & 228.690 & 26.443 \\
237.069 & 26.971 & 245.448 & 27.511 \\
253.828 & 28.062 & 262.207 & 28.625 \\
270.586 & 29.200 & 278.966 & 29.788 \\
287.345 & 30.387 & 295.724 & 30.999 \\
304.103 & 31.623 & 312.483 & 32.260 \\
320.862 & 32.910 & 329.241 & 33.573 \\
337.621 & 34.250 & 346.000 & 34.939 \\
$($ Spline interpolation has been & used to generate the grid.) \\
\hline & & & \\
\hline
\end{tabular}

